\title{
Contribuição à gestão de riscos no processo de projeto de incorporadoras de médio porte
}

\author{
Contribution to risk management in the design process of \\ midsize construction companies
}

\section{Felipe de Souza Pinto Barreto \\ Paulo Roberto Pereira Andery}

\section{Resumo}

A

melhoria do processo de projeto de empresas construtoras leva à necessidade de introdução de mecanismos de gestão de riscos desde a fase de concepção dos empreendimentos. Nesse sentido, o artigo apresenta os resultados de pesquisa que teve como objetivo mapear o processo de projeto de empresas incorporadoras e construtoras de médio porte, identificando quais são as atividades no processo de projeto que impactam na gestão de riscos. A análise do processo de projeto usou como referencial as ações propostas na ISO31000 e no PMBOK. Uma vez mapeadas as atividades associadas à gestão de riscos, as mesmas foram hierarquizadas, do ponto de vista de seu impacto na redução de riscos, a partir dos critérios de priorização estabelecidos pelo Project Definition Rating Index - PDRI, do CII. A pesquisa se desenvolveu por meio de estudos de caso múltiplos, realizados em três empresas de médio porte. As fontes de evidências envolveram análise de documentos e projetos, entrevistas e observação direta de rotinas de trabalho. Os resultados apontam para o fato de que as empresas não possuem mecanismos adequados para a gestão de riscos na fase de concepção, e esses mecanismos concentram-se, sobretudo, na etapa de análise de viabilidade econômica dos empreendimentos. A explicitação das ações envolvidas com a gestão de riscos pode ser um primeiro passo para que as empresas implementem modelos de gestão de riscos nos estágios iniciais de desenvolvimento dos empreendimentos.

Palavras-chave: Gestão do processo de projeto. Gestão de riscos. Incorporação imobiliária.

Felipe de Souza Pinto Barreto Universidade Federal de Minas Gerais Belo Horizonte - MG - Brasil

Paulo Roberto Pereira Andery Universidade Federal de Minas Gerais Belo Horizonte - MG - Brasil

Recebido em 13/03/15 Aceito em 17/07/15

\begin{abstract}
In order to improve the design processes of building companies it is necessary to introduce risk management mechanisms at an earlier stage of construction projects. This paper presents the results of a research study aimed at mapping the design process of midsize construction companies, identifying which activities in the design process can be associated with risk management. The analysis used the conceptual framework outlined by ISO 31000 and PMBOK. The mapped activities were prioritized based on criteria set up by the Project Definition Rating Index, from Construction Industry Institute. The research method involved multiple case studies carried out in three medium-sized real estate companies. The sources of evidence included documents, design analysis, interviews and direct observation of work routines. The results point to the fact that companies do not have adequate mechanisms for risk management at the design stage. Risk analysis is mainly concentrated on economic feasibility evaluations. The deployment of actions involved in risk management can be a first step for companies to implement risk management models at earlier stages of construction projects.
\end{abstract}

Keywords: Design management. Risk management. Real estate. 


\section{Introdução}

Nos últimos anos o mercado imobiliário brasileiro, particularmente no setor de incorporações voltadas à promoção de edifícios residenciais e comerciais, tem passado por significativas transformações. Um dos vetores de transformação diz respeito a mudanças no quadro macroeconômico, a implementação de políticas de incentivo a esse mercado e a criação de uma estrutura jurídica que favoreceu o setor. São medidas que criaram um contexto, externo às empresas, de incentivo à promoção dos empreendimentos, que geraram uma expansão do setor maior ou menor, segundo os casos (CÂMARA..., 2015). Por outro lado, e em parte como resposta a essa conjuntura de mercado, muitas empresas aprimoraram seus sistemas de gestão da produção, como estratégia de melhoria de sua competitividade (CARDOSO et al., 2012).

Esse contexto competitivo da indústria da construção leva a um crescente interesse por melhores resultados em termos de garantia da qualidade das edificações. A dimensão de desempenho ganha importância, bem como garantia do custo e prazos de entrega dos empreendimentos. Isso passa a estar diretamente associado à melhoria da etapa de concepção e projeto.

O mesmo caminho foi seguido em países mais desenvolvidos: significativa parcela das empresas investiu em melhorias na gestão do processo de projeto (PRINS; OWEN, 2010; EMMIT; DEMIAN; ALHARBI, 2011), implementando procedimentos sistêmicos, associados ou não aos sistemas de gestão da qualidade, abrangendo desde a fase de concepção até a orçamentação e planejamento das operações nos canteiros de obra, ou seja, nas atividades "pré-obra".

Dessa forma, passa a ser importante avaliar o grau de maturidade da fase de concepção e desenvolvimento de projetos, bem como explicitar mecanismos de identificação e tratamento de riscos existentes ou que possam ser implementados, já que o esforço por aperfeiçoar o processo de projeto impacta diretamente na redução do nível de incerteza e risco ao longo do ciclo de vida dos empreendimentos (MARQUES; STARLING; ANDERY, 2013; AZEVEDO, 2013; ROSTAMI et al., 2015).

Em outras palavras, tanto nas práticas de mercado quanto do ponto de vista acadêmico revela-se a importância de diagnosticar e melhorar as práticas de gestão de riscos no processo de projeto de empresas voltadas à incorporação imobiliária.

É nesse contexto que se insere o presente trabalho, que realiza um estudo exploratório que identifica as práticas de gestão de riscos encontradas no processo de concepção e desenvolvimento de projetos de empresas incorporadoras e construtoras de médio porte.

Parte-se da hipótese de que tais empresas têm implícitas em seus processos, muitas vezes de maneira informal, atividades que auxiliam na identificação e tratamento de riscos. A literatura aponta para o fato de que diagnosticar e explicitar os processos de identificação e análise de riscos é passo prévio para a introdução de mecanismos sistêmicos e formais de gestão de riscos (SABADA et al., 2014; GEOFREY; HALCROW, 1996). É com essa perspectiva que foi desenvolvido o presente trabalho.

A partir desse passo, planos e ação podem ser elaborados para a melhoria da gestão do processo de concepção e projeto dos empreendimentos, com melhor definição do objetivo das atividades de projeto e a introdução de métodos e ferramentas de coordenação que reduzam a incerteza principalmente quanto ao custo, prazo e escopo dos empreendimentos (CHO; GIBSON, 2001).

Essa parece ser uma lacuna no que diz respeito aos meios acadêmicos brasileiros, nos quais o processo de projeto de construtoras de médio porte, sob a ótica da gestão de ricos, foi ainda pouco investigado.

A pesquisa compreendeu estudo de caso múltiplo, realizado em três empresas nas quais as atividades que impactam na gestão de riscos foram identificadas à luz das diretrizes encontradas em referências consagradas, em particular a norma ISO 31000 (ABNT, 2009) e o guia de melhores práticas de análise de riscos proposto no Project Management Body of Knowledge (PMBOK) (PROJECT..., 2013).

$\mathrm{Na}$ sequência apresenta-se um breve referencial teórico, o método de pesquisa é delineado, e são expostos os resultados dos estudos de caso.

\section{Breve referencial teórico}

Gradativamente vem crescendo a percepção sobre a importância da gestão de riscos na fase de projeto, em função de um mercado que, também internacionalmente, é caracterizado pela restrição de recursos, cronogramas apertados, aumento da competição e redução das margens de lucro. Esse aspecto torna-se particularmente crítico no caso de empresas construtoras de médio porte (ROSTAMI et al., 2015). 
De fato, estudos recentes apontam para a constatação de que aumenta a complexidade dos projetos e a exposição a riscos. Ainda que a definição das causas de incerteza varie de acordo com a percepção dos agentes - promotores, projetistas ou empreiteiros -, há concordância no fato de que a redução do risco está associada à melhoria do processo de projeto, em especial à própria gestão dos riscos nessa fase (MC GRAW HILL, 2014).

Nessa direção, várias pesquisas apontam para o fato de que a identificação e tratamento de riscos na fase de projeto são fundamentais para a garantia de custos, prazos e escopo dos empreendimentos (MC GRAW-HILL, 2014). Essa constatação não é recente no âmbito acadêmico: vejam-se, por exemplo, os trabalhos de Huffman (2002) e Edwards e Bowen (1998).

Outras pesquisas sugerem que a forma como os riscos são identificados nas fases iniciais dos empreendimentos terá grande impacto na maneira como eles serão mitigados ou monitorados na fase de construção e operação (CHAPMAN, 2001).

Observa-se nas práticas de mercado uma situação em si mesma contraditória. Por um lado, vai crescendo a consciência a respeito da importância da diminuição do nível de incerteza na concepção e projeto dos empreendimentos. Por outro lado, pouco se pratica nesse sentido, o que parece ser também uma realidade em âmbito internacional, em especial quando se trata de empresas de pequeno e médio porte (MC GRAW-HILL, 2014; TAROUN, 2014). Também no âmbito acadêmico ainda são poucos os trabalhos que abordam a gestão de riscos no processo de projeto, ainda que seja essa uma preocupação crescente (AZEVEDO, 2013).

No mercado de desenvolvimento imobiliário brasileiro a análise de riscos quase sempre concentra a atenção em aspectos econômicofinanceiros do negócio, incluindo a análise de viabilidade econômica e de conjunturas de mercado externas à empresa (BUZI; JUNGLES; AVILA, 2012; AZEVEDO, 2013), sem que maior atenção seja dada à fase de concepção.

Seguindo uma tendência da literatura recente, atividades associadas à gestão de riscos podem ser identificadas a partir do referencial normativo da ISO 31000 (ABNT, 2009), que apresenta semelhança com os outros métodos de gestão de riscos, como o apresentado pelo PMBOK (PROJECT..., 2013), ou ainda pelo Practice Standard for Project Risk Management (PROJECT..., 2009).
Estudo mostrando que as estruturas conceituais desses sistemas são semelhantes é apresentado em Azevedo (2013), o qual, por questões de brevidade, foge ao escopo do presente trabalho. Essas referências normativas estão em harmonia com diretrizes propostas nos meios acadêmicos, como é o caso de Sabada et al. (2014).

A ISO 31000 (ABNT, 2009) fornece uma estrutura conceitual para a análise de riscos que leva em conta as políticas e práticas da empresa - e passa a ser incorporada a elas -, tanto em nível estratégico como operacional. Essa estrutura conceitual da norma, desdobrada em requisitos, ações e processos, implicará a introdução de mecanismos de melhoria contínua nos processos de gestão. A análise de riscos considera ao mesmo tempo o ambiente do projeto e o ambiente empresarial (SOUZA; ALMEIDA; DIAS, 2012; BHARANTY; MCSHANE, 2014).

Por outro lado, serve como referência para que os processos de gestão de riscos sejam identificados e aprimorados (PURDY, 2010).

Os processos de análise de riscos descritos no PMBOK têm uma estrutura conceitual semelhante, e uma comparação nesse sentido é apresentada em Azevedo (2013).

Uma vez identificados riscos nas distintas atividades do processo de projeto, é preciso definir uma forma de hierarquizá-los em função de seu impacto no resultado do empreendimento, em termos de escopo, custo ou prazo, e da probabilidade, seja ela qualitativa ou quantitativa, de ocorrência do evento incerto. Uma das formas específicas de hierarquização de riscos foi proposta por meio do Project Definition Rating Index (PDRI), do Construction Industry Institute (CONSTRUCTION..., 2008; CHO; GIBSON, 2001).

O PDRI configura-se como ferramenta para avaliação do nível de detalhamento (ou grau de definição de atividades do processo de projeto), associando esse nível de definição ao nível de incerteza e risco no desempenho do projeto. Nesse sentido, identifica e descreve elementos críticos que impactam no nível de risco. Supõe que determinada atividade, se não conduzida propriamente, tendo baixo nível de detalhamento (ou cumprimento de seu escopo), impactará no aumento do risco.

Para cada atividade é estabelecido um peso máximo, que determina o impacto dessa atividade em termos de risco, supondo que não foi adequadamente realizada (CONSTRUCTION..., 2008). Assume-se, portanto, que, se o nível de cumprimento do escopo da atividade é baixo, o 
impacto no risco será tanto maior quanto maior for o peso atribuído.

O total de atividades, com seus pesos máximos somados, implica um valor máximo de 1.000 pontos, correspondentes à pior situação possível, ou seja, máxima exposição ao risco. Quanto menores os pesos das atividades, menor será a pontuação total, o que implica melhor definição do projeto e menor exposição ao risco.

Dessa forma, o PDRI pode ser utilizado como parâmetro balizador de atividades no processo de projeto que impactam mais ou menos no risco de insucesso dos empreendimentos.

Diversos trabalhos na literatura internacional correlacionaram a aplicação do PDRI com o nível de risco e o insucesso do projeto, traduzido em termos de aumento de custos e/ou do prazo de execução. Mais recentemente, Fageha e Aibinu (2014) associaram a aplicação do PDRI com atividades críticas de projeto, sugerindo que a ferramenta permite priorizar atividades que precisam ter melhor nível de definição ou detalhe.

Uma lista de atividades do PDRI com seus respectivos pesos em um total de 1.000 pontos é apresentada na Tabela 1 . Na tabela são também indicados os códigos associados a cada atividade, conforme explicitado no PDRI (CONSTRUCTION..., 2008).

\section{Método de pesquisa}

Como indicado anteriormente, os estudos de caso buscaram mapear o processo de projeto das empresas e relacionar que atividades impactam na identificação e tratamento de riscos. As atividades do processo de projeto, uma vez mapeadas, são comparadas com as ações indicadas na estrutura conceitual da ISO 31000 e do PMBOK.

Assume-se que a etapa de concepção e projeto englobe atividades que vão da escolha do terreno e formulação do conceito do empreendimento até as atividades posteriores ao desenvolvimento dos projetos, como orçamentação e planejamento da execução, ou seja, o conjunto de atividades anteriores à fase de execução nos canteiros de obra.

O recorte de análise é o de empresas incorporadoras e construtoras de médio porte, de acordo com a classificação do Sebrae.

Partiu-se da suposição de que o processo de projeto de empresas incorporadoras de pequeno e médio porte com frequência não é formalmente estruturado, e maior parte das ações que impactam na identificação e gestão de riscos não é desenvolvida de maneira formal e estruturada.

O método do estudo de caso seguiu os procedimentos sugeridos por Yin (2013).

De maneira sintética, as etapas da pesquisa foram:

(a) revisão bibliográfica, propiciando estrutura conceitual para a formulação do protocolo de estudo de caso e identificação de ações, nas empresas, relacionáveis com a gestão de riscos;

(b) definição dos critérios para seleção das empresas e seleção das três empresas estudadas;

(c) condução de estudo de caso piloto na Empresa A, conforme indicado em Barreto (2015);

(d) validação do protocolo de estudo de caso, que envolveu pequenas alterações nos questionários conduzidos com os sócios proprietários e os diretores responsáveis pela área de incorporação e desenvolvimento de projetos;

(e) coleta de dados nas empresas B e C;

(f) elaboração de análise dos processos de gestão de riscos em cada empresa; e

(g) triangulação dos dados e análise conjunta.

As empresas objeto do estudo de caso foram selecionadas com base nos seguintes critérios:

(a) ser empresas de médio porte;

(b) disponibilidade para participar da pesquisa, incluindo o acesso às fontes de evidências, possibilidade de entrevistar dirigentes e observar rotinas internas de trabalho; e

(c) existência de um processo de projeto que, ainda que não necessariamente formalizado, seja essencialmente o mesmo nos vários empreendimentos, de forma que a análise de atividades associadas à gestão de riscos seja representativa da realidade da empresa.

O protocolo dos estudos de caso é detalhadamente descrito em Barreto (2015).

As fontes de evidências englobaram planilhas e documentos de análise de viabilidade econômicofinanceira, técnica e jurídica; fluxogramas de processos; acesso a sistemas de gestão empresarial e de documentos; manuais dos sistemas de gestão da qualidade (nas Empresas A e B); relatórios técnicos e gerenciais; projetos e seus documentos associados, em suas várias fases; procedimentos de aquisição de terrenos; relatórios de desempenho econômico-financeiro, entre outros documentos. Nas Empresas A e B foram acompanhadas rotinas de trabalho.

74 Barreto, F. de S. P.; Andery, P. R. P. 
Tabela 1 - Relação de elementos do processo de projeto com seus respetivos pesos

\begin{tabular}{|c|c|}
\hline \multicolumn{2}{|l|}{ SEÇÃO I - BASE DA ELABORAÇÃO DO PROJETO } \\
\hline \multirow{2}{*}{$\begin{array}{l}\text { CATEGORIA } \\
\text { Elemento }\end{array}$} & Pontuação \\
\hline & Max \\
\hline \multicolumn{2}{|l|}{ A. ESTRATÉGIA DE NEGÓCIOS } \\
\hline A1 Uso do edifício & 44 \\
\hline A2 Justificativa de negócio & 27 \\
\hline A3 Plano de negócios & 26 \\
\hline A4 Análise econômica & 21 \\
\hline A5 Requisitos de instalação & 31 \\
\hline A6 Expansão futura & 22 \\
\hline A7 Considerações do mercado local & 28 \\
\hline A8 Declaração dos objetivos do projeto & 15 \\
\hline CATEGORIA A TOTAL & 214 \\
\hline \multicolumn{2}{|l|}{ B. FILOSOFIAS DO PROPRIETÁRIO } \\
\hline B1 Filosofia de confiabilidade & 18 \\
\hline B2 Filosofia de manutanção & 16 \\
\hline B3 Filosofia de operação & 15 \\
\hline \multirow[t]{2}{*}{ B4 Filosogia de projeto } & 19 \\
\hline & 68 \\
\hline \multicolumn{2}{|l|}{ C. REQUISITOS DO PROJETO } \\
\hline C1 Processo de análise de valor & 19 \\
\hline C2 Critérios de elaboração do projeto & 24 \\
\hline C3 Avaliação das instalações existentes & 24 \\
\hline C4 Escopa da visão geral de trabalho & 17 \\
\hline C5 Programação do cronograma do projeto & 20 \\
\hline C6 Estimativa de custo do projeto & 27 \\
\hline CATEGORIA C TOTAL & 131 \\
\hline SEÇÃO I TOTAL & 413 \\
\hline \multicolumn{2}{|l|}{ SEÇÃO II - E } \\
\hline $\begin{array}{l}\text { CATEGORIA } \\
\text { Elemento }\end{array}$ & Pontuação \\
\hline Elemento & Max \\
\hline \multicolumn{2}{|l|}{ D. INFORMAÇÕES DO LOCAL } \\
\hline D1 Disposição do local & 14 \\
\hline D2 Vistoria e investigação local & 14 \\
\hline D3 Informações construtivas e geotécnicas & 19 \\
\hline D4 Exigências regulatórias governamentais & 14 \\
\hline D5 Avaliação ambiental & 16 \\
\hline D6 Serviços públicos com condições de fornecimento & 13 \\
\hline D7 Considerações de proteção à vida para o local selecionado & 8 \\
\hline D8 Requisitos de tratamento especial de água e de resíduos & 11 \\
\hline CATEGORIA D TOTAL & 109 \\
\hline E. PLANEJAMENTO DA CONSTRUÇÃO & \\
\hline E1 Declaração de programa & 16 \\
\hline E2 Lista resumo do espaço doedifício & 21 \\
\hline E3 $\quad$ Diagramas gerais de adjacência & 10 \\
\hline E4 Diagrama de empilhamento & 13 \\
\hline E5 Crescimento e desenvolvimento nas fases futuras & 15 \\
\hline E6 Exigências de espaço aberto e circulação & 13 \\
\hline E7 Diagramas da relação funcional /ambiente por ambiente & 10 \\
\hline E8 Requisitos de capacidade de carga, descarga e armazenamento & 8 \\
\hline E9 Requisitos de transporte & 9 \\
\hline E10 Acabamento da construção & 15 \\
\hline E11 Informações do ambiente & 13 \\
\hline E12 Mobiliário, equipamentos e embutidos & 14 \\
\hline E13 Tratamento de janela & 5 \\
\hline CATEGORIA E TOTAL & 162 \\
\hline F. PARÂMETROS PARA ELABORAÇÃO DO PROJETO / CONSTRUÇÃO & \\
\hline F1 Projeto construtivo local & 14 \\
\hline $\begin{array}{ll}\text { F2 } & \text { Projeto arquitetônico } \\
\end{array}$ & 22 \\
\hline $\begin{array}{ll}\text { F3 } & \text { Projeto estrutural } \\
\end{array}$ & 18 \\
\hline F4 Projeto mecânico & 20 \\
\hline F5 Projeto elétrico & 15 \\
\hline F6 Requisitos de proteção à vida no edifício & 10 \\
\hline $\begin{array}{ll}\text { F7 } & \text { Análise de construtivilidade } \\
\end{array}$ & 14 \\
\hline F8 Sofisticação tecnológica & 9 \\
\hline CATEGORIA F TOTAL & 122 \\
\hline G. EQUIPAMENTOS & \\
\hline G1 Lista de equipamentos & 15 \\
\hline G2 Desenhos da posição de equipamentos & 10 \\
\hline G3 Requisitos de uso de equipamentos & 11 \\
\hline CATEGORIA G TOTAL & 36 \\
\hline SEÇÃO ॥ TOTAL & 429 \\
\hline
\end{tabular}


Em todas as empresas foram conduzidas entrevistas semiestruturadas com os sócios proprietários e coordenadores de projetos. Essas entrevistas buscaram:

(a) caracterizar a empresa quanto a porte, perfil dos empreendimentos, faturamento, organograma e estrutura funcional, sistemas de gestão empresarial e gestão de operações;

(b) mapeamento do processo de projeto; e

(c) atividades e ferramentas usadas no processo de projeto.

Em cada empresa a análise das fontes de evidências objetivou o estudo sobre três aspectos; o primeiro, de caracterização da empresa, incluindo aqui estrutura organizacional, perfil dos empreendimentos, faturamento, etc.

O segundo aspecto foi o de caracterização do processo de projeto, denominado "macroprocesso de projeto". Esse processo foi desdobrado em etapas, e essas em atividades, resumidamente apresentadas na próxima seção.

O terceiro aspecto foi a associação das atividades de projeto com os mecanismos de identificação e tratamento de riscos, conforme indicados na ISO 31000 e no PMBOK.

\section{Estudo de caso múltiplo: análise do processo de projeto e a gestão de riscos}

\section{Caracterização das empresas}

As empresas, identificadas como "A", "B" e "C", são empresas incorporadoras e construtoras de médio porte.

Duas das empresas estudadas (Empresas B e C) têm um perfil gerencial próximo ao de empresas de pequeno porte, como caracterizado em Bicalho (2009), ou seja:

(a) estrutura organizacional flexível e não formalizada. Ocorre concentração do poder de decisão sobre o proprietário; (b) ausência de um planejamento estratégico;

(c) dificuldades na implantação de SGQ

(Sistemas de Gestão da Qualidade), quando existente;

(d) funcionários desempenham funções variadas dentro da empresa; e

(e) inexistência de mecanismos formais de controle de processos.

Com relação ao perfil das empresas, a Tabela 2, abaixo, apresenta uma síntese das características principais.

A Empresa A, embora tenha porte similar em termos de número de empreendimentos $\mathrm{e}$ funcionários, possui características distintas das outras duas. É uma empresa que, do ponto de vista gerencial, é mais evoluída. A partir das entrevistas e das outras fontes de evidências mencionadas acima foi possível identificar que essa empresa possui processos padronizados e incorporados a sua rotina.

Essa explicitação e padronização de processos diz respeito a vários âmbitos da gestão da produção. A empresa contratou em agosto de 2014 uma consultoria que promoveu a estruturação do processo de projeto, incluindo o estudo de viabilidade, desenvolvimento das várias etapas do projeto e orçamentação. Na gestão de obras, a utilização de software de gestão empresarial permite a integração entre as funções de planejamento, suprimentos, apropriação de custos e controle da produção, entre outros aspectos. A implementação do sistema de gestão da qualidade (Sistema de Avaliação de Conformidade - SIAC e ISO 9001) encontra-se consolidada, com rotinas aplicadas a todos os empreendimentos.

No âmbito da gestão empresarial, a empresa definiu ao longo do tempo processos de gestão que não são objeto de estudo deste trabalho - e criou indicadores de desempenho empresarial.

A empresa conta com consultoria de Recursos Humanos e consultoria permanente de melhoria da gestão empresarial.

Tabela 2 - Características das empresas estudadas

\begin{tabular}{l|c|c|c} 
& Empresa A & Empresa B & Empresa C \\
\hline Local de Atuação & Belo Horizonte & Belo Horizonte & Campos dos Goytacazes \\
\hline Funcionários & 450 & 160 & 400 \\
\hline Mercado de Atuação & Edifícios habitacionais & $\begin{array}{c}\text { Edifícios habitacionais / } \\
\text { Comerciais }\end{array}$ & $\begin{array}{c}\text { Edifícios habitacionais / } \\
\text { Comerciais }\end{array}$ \\
\hline Tipologia Média do Produto & $\begin{array}{c}\text { Apartamento 2 } \\
\text { dormitórios }\end{array}$ & $\begin{array}{c}\text { Não existe tipologia } \\
\text { específica }\end{array}$ & Apartamento 3 dormitórios \\
\hline Empreendimentos Lançados & 10 & 12 & 11 \\
\hline Unidades Incorporadas & 1.581 & 450 & 340 \\
\hline Padrão Construtivo & Baixo & Normal & Normal \\
\hline Sistema de Gestão & ISO 9001 e PBQP-H & ISO 9001 e PBQP-H & Não possui \\
\hline
\end{tabular}

76 Barreto, F. de S. P.; Andery, P. R. P. 
Suas metas de operação são revistas trimestralmente, e o planejamento estratégico é discutido semestralmente pelo conselho consultivo da empresa, formado por seus diretores e especialistas convidados.

Essas características parecem estar ligadas ao perfil empresarial de seu diretor presidente, executivo de experiência que exerceu funções gerenciais em empresas de grande porte.

A Empresa B encontrava-se durante o desenvolvimento da pesquisa (segundo semestre de 2014) em processo de transição, passando de uma situação na qual predominam procedimentos não formalizados para outra em que os processos passam a ser formalmente definidos e padronizados. Essa transição é evidenciada principalmente pela maior preocupação com a implantação efetiva do Sistema de Gestão da Qualidade nos principais processos gerenciais da empresa.

No entanto, a Empresa B ainda carece de processos que auxiliem a tomada de decisão e o controle efetivo das atividades em desenvolvimento. Isso se deve principalmente a sua característica de empresa familiar, em que importantes decisões são tomadas sem um processo burocrático e documentos de formalização. O cronograma e o orçamento, quando elaborados, não contemplam a fase de concepção.

A Empresa C é a que tem menor nível de formalidade em seus processos. A ausência da certificação na ISO 91000 e no Sistema de Avaliação de Conformidade do PBQP-H é justificada pela empresa pelo fato de que não necessita de mecanismos de financiamento em órgãos que fazem essa exigência. A própria justificativa - obtida mediante depoimento de um dos sócios diretores - parece mostrar uma visão estreita que seus sócios diretores têm a respeito dos sistemas de gestão da qualidade.

\section{Caracterização do processo de projeto}

Os macrofluxos de projeto, em suas etapas e atividades, são similares nas empresas estudadas. $\mathrm{O}$ que os distingue são os procedimentos utilizados em cada caso, em seu nível de rigor e detalhe.

Algumas das atividades que parecem impactar mais na gestão de riscos, particularmente nas etapas iniciais de definição do produto e análise de viabilidade, são executadas nas três empresas, com maior ou menor efetividade de acordo com cada uma, ou seja, em parte dos processos, não obstante o perfil diferente das empresas, há similaridade entre ações orientadas à identificação e ao tratamento de riscos.

Foi identificado um macrofluxo de projeto que pode ser subdividido em seis etapas, cada uma delas desdobradas em conjuntos de processos.

A Empresa A é a única que aplica as seis, ilustradas na Figura 1, a seguir.

Diferentemente das Empresas B e C, a Empresa A aplica em seu processo dois gates de avaliação e validação.

$\mathrm{O}$ primeiro gate tem o objetivo de avaliar as opções de concepção dos empreendimentos e escolher a mais viável ou de maior interesse para a empresa. Já o segundo gate visa quantificar a atratividade da opção, com base em indicadores econômico-financeiros. São calculados o valor presente líquido (VPL) e a taxa interna de retorno (TIR), a partir dos quais o empreendedor fundamenta sua decisão de empreender ou não, mediante simulação de vários cenários para fluxo de caixa, velocidade de vendas e custos das obras.

No entanto, apenas no primeiro gate é feita uma avaliação das distintas opções de terrenos disponíveis e da tipologia do empreendimento. O segundo gate assume que uma opção já foi selecionada e faz a análise da atratividade. Dessa forma, perde-se a capacidade de mitigar os riscos e potencializar outras oportunidades, através da seleção de uma opção de empreendimento menos arriscada e com maior potencial de rentabilidade, uma vez que as opções são descartadas prematuramente.

$\mathrm{Na}$ fase de "prospecção de terrenos" observa-se que a empresa trabalha com um banco de dados de terrenos e estudos de viabilidades anteriores, que permite balizar futuras análises. Por outro lado, ao contrário das demais empresas, realiza pesquisas sobre terrenos de maneira contínua, levantando oportunidades futuras e fazendo um registro histórico de fatores condicionantes da viabilidade dos empreendimentos.

O macrofluxo de projeto das Empresas B e C são mais similares entre si. Ambas possuem apenas um gate para verificação e aprovação do empreendimento. A tomada de decisão para aquisição ou permuta do terreno é feita nesse momento.

O macrofluxo da Empresa B é apresentado na sequência (Figura 2). 
Figura 1 - Macrofluxo de projeto da Empresa A

\section{EMPRESA A}

MACRO FLUXO DE PROJETO

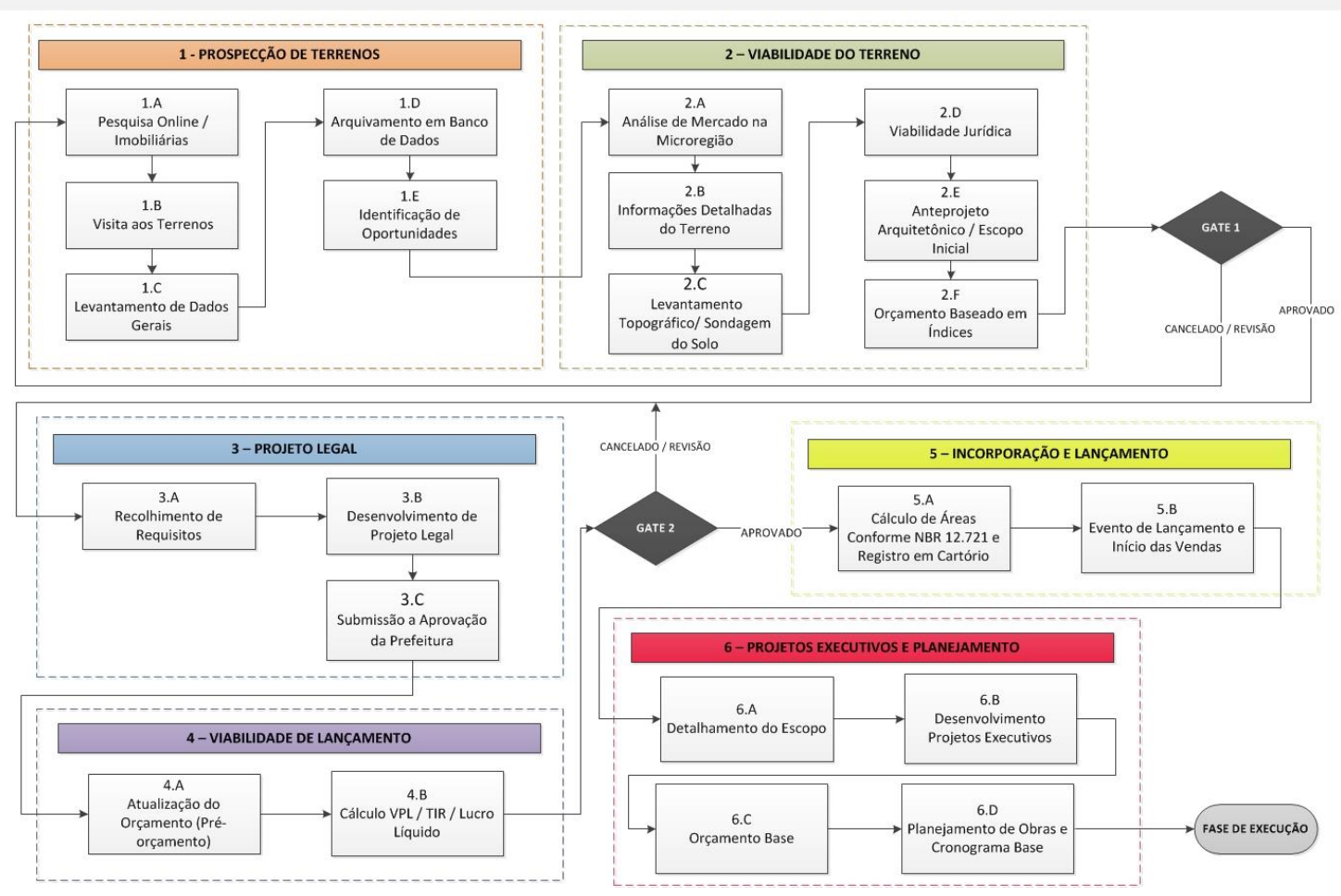

Figura 2 - Macrofluxo de projeto da Empresa B

\section{EMPRESA B}

\section{MICROPROCESSO DE CONCEPÇÃO DE EMPREENDIMENTOS}
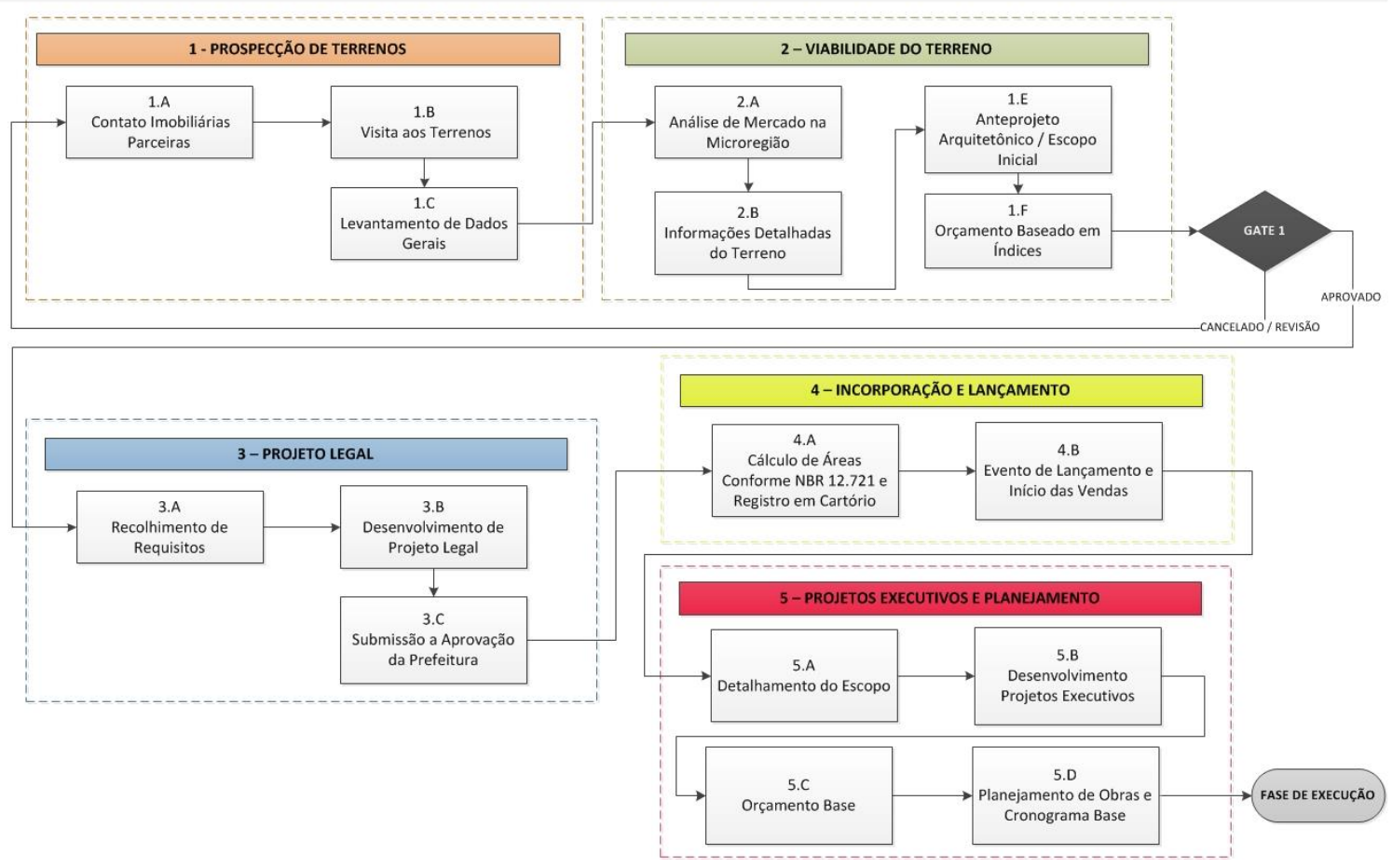

78 Barreto, F. de S. P.; Andery, P. R. P. 
Nesse caso, a tomada de decisão é suportada por um baixo nível de informações e uma análise financeira pouco precisa. Esta empresa não desenvolve atividades importantes como a prospecção inicial do solo e a análise de viabilidade jurídica do terreno. Ao contrário da Empresa A, não há uma fase específica para análise da viabilidade do lançamento do empreendimento.

A ausência desses processos acarreta maior grau de incerteza, que pode levar a uma tomada de decisão errada, o que pode vir a comprometer não só o sucesso do empreendimento mas também a saúde financeira da empresa.

A Figura 3 apresenta o macrofluxo de projeto para a Empresa C, similar ao apresentado na figura anterior. Apesar de apresentar menor nível de formalidade em seus processos, o estudo de viabilidade na Empresa $C$ parece ser mais criterioso que o da Empresa $\mathrm{B}$, incluindo levantamentos topográficos e sondagens de terreno como condição para análise de viabilidade. O tratamento de riscos é ainda mais bem explicitado com a presença de uma fase de análise jurídica de potenciais terrenos a ser adquiridos.

Observa-se que as Empresas $\mathrm{B}$ e $\mathrm{C}$ não desenvolvem a fase de viabilidade de lançamento, que consiste numa atualização do orçamento e em uma análise financeira mais refinada e precisa, que é realizada nos estudos iniciais de análise de viabilidade.

\section{Identificação das atividades de gestão de riscos no processo de projeto}

Uma vez mapeadas, as atividades distribuídas nas distintas etapas do macrofluxo de projeto foram relacionadas a ações voltadas à gestão de riscos, conforme referencial da ISO 31000 e PMBOK. Um ou mais processos de gestão de riscos podem ser relacionados às atividades de projeto, de forma que os requisitos do referencial possam ser total ou parcialmente cumpridos.

A Tabela 3 apresenta essas ações, com descrição resumida de cada item, por razões de brevidade. Uma análise detalhada das ações para cada processo em cada empresa é apresentada em Barreto (2015).

Observa-se que as atividades associadas à gestão de riscos que as empresas melhor realizam, e que são mais semelhantes entre si quando de uma comparação entre as empresas, são aquelas orientadas especificamente ao processo de incorporação, em particular à análise de viabilidade do terreno e à análise jurídica. São inerentes à atividade de incorporação, e menos dependentes do desenvolvimento da atividade projetual.

Figura 3 - Macrofluxo de projeto da Empresa C

\section{EMPRESA C}

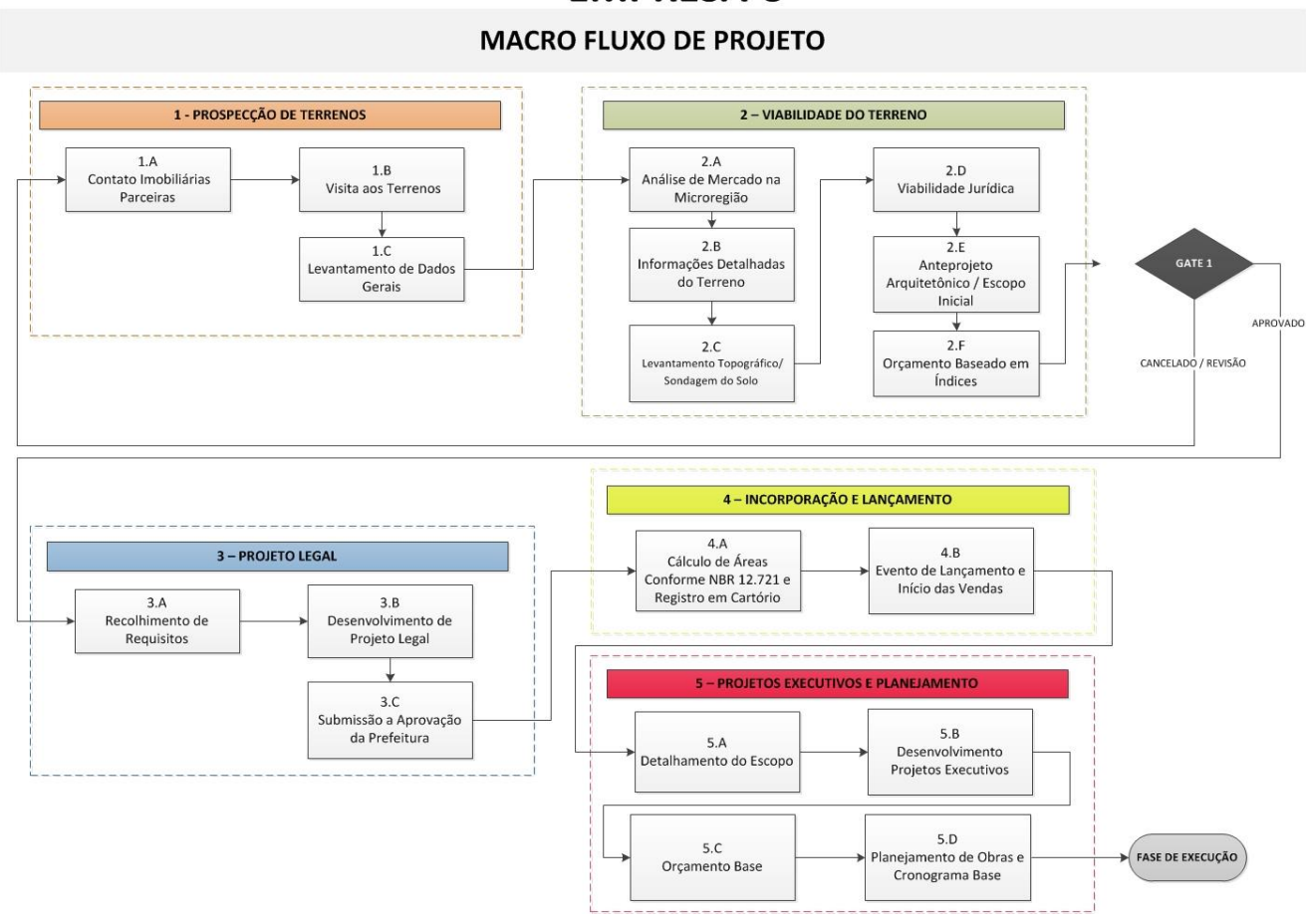


Tabela 3 - Contribuição de cada atividade de concepção para a Gestão dos Riscos

\begin{tabular}{|c|c|c|c|c|c|c|}
\hline Etapas & Atividades & Empresa A & Empresa B & Empresa C & Elementos de Gestão de Riscos & $\begin{array}{l}\text { Processos } \\
\text { ISO } 31.000\end{array}$ \\
\hline \multirow{5}{*}{$\begin{array}{l}1 \text { - Prospeção de } \\
\text { Terrenos }\end{array}$} & 1.A - Pesquisas Online/ Imobiliárias & & & & $\begin{array}{l}\text { Identificar diversas opções a fim de mitigar a chance de } \\
\text { estar escolhendo um terreno de menor atratividade se } \\
\text { comparado a outros disponíveis no mercado }\end{array}$ & $\begin{array}{l}\text { II) Identificação dos Riscos } \\
\text { II) Análise dos Riscos } \\
\text { III) Avaliação dos Riscos }\end{array}$ \\
\hline & 1.B - Visita aos Terrenos & & & & \begin{tabular}{|l|} 
Identificação e análise de riscos relacionados a \\
infraestrutura, logística, vizinhança, topografia, geotecnia
\end{tabular} & $\begin{array}{l}\text { I) Identificação dos Riscos } \\
\text { II) Análise dos Riscos } \\
\text { III) Avaliação dos Riscos }\end{array}$ \\
\hline & 1.C - Levantamento de Dados Gerais do Terreno & & & & $\begin{array}{l}\text { Através do levantamento de dados podem ser identificado } \\
\text { outros riscos com impacto importante no sucesso do } \\
\text { empreendimento. }\end{array}$ & (d) Identificação dos Riscos \\
\hline & 1.D - Arquivamento em Banco de Dados & & & & $\begin{array}{l}\text { Facilita a comparação com outros terrenos pesquisadoś } \\
\text { uma forma de análisar os riscos }\end{array}$ & \begin{tabular}{|l|} 
II) Análise dos Riscos \\
III) Avaliação dos Riscos
\end{tabular} \\
\hline & 1.E - Identificação de Oportunidades & & & & $\begin{array}{l}\text { Análise de riscos para escolha da melhor oportunidade } \\
\text { disponível no mercado }\end{array}$ & $\begin{array}{l}\text { I) Análise dos Riscos } \\
\text { II) Avaliação dos Riscos }\end{array}$ \\
\hline \multirow{7}{*}{$\begin{array}{l}2 \text { - Viabilidade do } \\
\text { Terreno }\end{array}$} & 2.A - Análise de Mercado na Microrregião & & & & $\begin{array}{l}\text { Identificação de riscos relacionados à concorrência e a } \\
\text { melhor opção construtiva para o empreendimento }\end{array}$ & $\begin{array}{l}\text { I) Identificação dos Riscos } \\
\text { II) Análise dos Riscos } \\
\text { III) Avaliação dos Riscos }\end{array}$ \\
\hline & 2.B - Informações Detalhadas do Terreno & & & & $\begin{array}{l}\text { Identificar riscos adicionais ou não mapeados na fase } \\
\text { anterior. }\end{array}$ & I) Tratamento dos Riscos \\
\hline & $\begin{array}{l}\text { 2.C - Levantamento Topográficol Sondagem do } \\
\text { Solo }\end{array}$ & & & & $\begin{array}{l}\text { Identificação de riscos relacionados a aumento de custos } \\
\text { com terraplanagem e fundaçôes Escolha de opção mais } \\
\text { viável. }\end{array}$ & $\begin{array}{l}\text { I) Identificação dos Riscos } \\
\text { II) Análise dos Riscos } \\
\text { III) Avaliação dos Riscos } \\
\text { IV) Tratamento dos Riscos }\end{array}$ \\
\hline & 2.D - Viabilidade Jurídica & & & & \begin{tabular}{|l|} 
Identificação de possíveis impedimentos jurílicos que \\
podem acarretar em atrasos na obra ou em casos extremo \\
na sua interdição e gerar medidas para soluciondos caso
\end{tabular} & $\begin{array}{l}\text { I) Identificação dos Riscos } \\
\text { II) Análise dos Riscos } \\
\text { III) Avaliação dos Riscos }\end{array}$ \\
\hline & & & & & & \\
\hline & 2.E - Anteprojeto Arquitetônico/ Escopo Inicial & & & & $\begin{array}{l}\text { Definição do escopo do projeto como forma de mitigação } \\
\text { dos riscos identificados. }\end{array}$ & $\begin{array}{l}\text { 1) Tratamento dos Riscos } \\
\text { II) Monitorar e Revisar }\end{array}$ \\
\hline & 2.F - Orçamento Baseado em Índices & & & & $\begin{array}{l}\text { Orçamento é baseado no projeto e nas incertezas } \\
\text { identificadas. }\end{array}$ & I) Avaliação dos Riscos \\
\hline \multirow{3}{*}{3 - Projeto Legal } & 3.A - Recolhimento de Requisitos & & & & $\begin{array}{l}\text { Identificar os requisitos corretamente garante uma } \\
\text { identificação ampla dos riscos Novos requisitos podem } \\
\text { surgir como forma de resposta a riscos já mapeados }\end{array}$ & $\begin{array}{l}\text { I) Identificação dos Riscos } \\
\text { II) Monitorar e Revisar }\end{array}$ \\
\hline & 3.B - Desenvolvimento de Projeto Legal & & & & $\begin{array}{l}\text { Apuração do escopo geral do empreendimento } \\
\text { considerando lista atualizada de requisitos e premissas. }\end{array}$ & $\begin{array}{l}\text { I) Tratamento dos Riscos } \\
\text { II) Monitorar e Revisar }\end{array}$ \\
\hline & 3.C - Submissão à Aprovação na Prefeitura & & & & $\begin{array}{l}\text { Controlar a ocorrência de riscos mapeados e identificação } \\
\text { de novos riscos. }\end{array}$ & I) Monitorar e Revisar \\
\hline \multirow{2}{*}{$\begin{array}{l}4 \text { - Viabilidade de } \\
\text { Lançamento }\end{array}$} & 4.A - Atualização do Orçamento(pré-orçamento) & & & & $\begin{array}{l}\text { dentificação de novos riscos e implementação de planos } \\
\text { de resposta a riscos já mapeados através do álaulo mais } \\
\text { apurado de contingenciamento e dos índicesquantitativos } \\
\text { e preços. }\end{array}$ & $\begin{array}{l}\text { I) Identificação dos Riscos } \\
\text { II) Tratamento dos Riscos }\end{array}$ \\
\hline & 4.B - Cálculo de VPL/ TIR / Lucro Líquido & & & & $\begin{array}{l}\text { O cálculo dos parâmetros econômicos traz um } \\
\text { embasamento numérico para a tomada de decisão }\end{array}$ & I) Avaliação dos Riscos \\
\hline \multirow{2}{*}{$\begin{array}{l}5 \text { - Incorporação e } \\
\text { Lançamento }\end{array}$} & $\begin{array}{l}\text { 5.A - Cálculo de Áreas conforme NBR12721 e } \\
\text { Registro em Cartório }\end{array}$ & & & & $\begin{array}{l}\text { Controla a implementação das ações e verifica a ocorrênc } \\
\text { de riscos mapeados e surgimento de novos riscos devido } \\
\text { a alterações de projeto não previstas }\end{array}$ & Monitorar e Revisar \\
\hline & 5.B - Evento de Lançamento e Início das Vendas & & & & $\begin{array}{l}\text { Controla a ocorrência de riscos mapeados e verifica o } \\
\text { aparecimento de novos riscos devido à variaçôs na } \\
\text { velocidade de vendas e às alteraçōes de projeto em relaçầ } \\
\text { ao material de propaganda. }\end{array}$ & I) Monitorar e Revisar \\
\hline \multirow{4}{*}{$\begin{array}{l}\text { 6- Projetos } \\
\text { Executivos e } \\
\text { Planejamento }\end{array}$} & 6.A - Detalhamento do Escopo & & & & $\begin{array}{l}\text { Implantação dos planos de resposta aos riscos já } \\
\text { identificados e surgimento de novos riscos }\end{array}$ & $\begin{array}{l}\text { I) Tratamento dos Riscos } \\
\text { II) Monitorar e Revisar }\end{array}$ \\
\hline & 6.B - Desenvolvimento de Projetos Executivos & & & & $\begin{array}{l}\text { Compatibilização de Projetos } \\
\text { Construtibilidade }\end{array}$ & $\begin{array}{l}\text { I) Tratamento dos Riscos } \\
\text { II) Monitorar e Revisar }\end{array}$ \\
\hline & 6.C - Orçamento Base & & & & Cálculo de Contingência & $\begin{array}{l}\text { I) Identificação dos Riscos } \\
\text { II) Tratamento dos Riscos }\end{array}$ \\
\hline & 6.D - Planejamento de Obras e Cronograma Base & & & & \begin{tabular}{|l|} 
Projeto de Canteiro Planejamento Logístico e \\
Interferências Previsão de ações que mitigam ou eliminan \\
riscos relacionados a baixa produtividade e retrabalho.
\end{tabular} & $\begin{array}{l}\text { I) Identificação dos Riscos } \\
\text { hiI) Tratamento dos Riscos }\end{array}$ \\
\hline & & \\
\hline & & \multicolumn{3}{|c|}{\begin{tabular}{l}
\multicolumn{1}{c}{ Legenda } \\
Realiza
\end{tabular}} & & \\
\hline & & & \multicolumn{2}{|c|}{\begin{tabular}{|l} 
Realiza parcialmente \\
Não realiza
\end{tabular}} & & \\
\hline
\end{tabular}

Mesmo em termos de viabilidade econômicofinanceira, fator mais bem identificado nas empresas como de impacto na gestão de riscos, as Empresas B e C realizam uma análise simplificada, sequer se pautando em parâmetros consagrados em gestão, como VPL ou TIR.

No conjunto de ações orientadas a gestão de riscos, relacionadas na Tabela 3 , observa-se que a Empresa A tem melhor desempenho. Esse fato aponta para duas possíveis causas.
Em primeiro lugar, a Empresa A conta com um sistema estruturado de gestão empresarial, que inclusive utiliza softwares integrados de gestão que permitem a extração de indicadores de desempenho empresarial ou de simulação de desempenho dos empreendimentos, e que são analisados pelo conselho consultivo da empresa. Em outras palavras, ainda que os processos sejam semelhantes aos executados nas outras empresas, o nível de análise é mais rigoroso, como decorrência 
de a empresa ser mais bem estruturada do ponto de vista gerencial.

Por outro lado, a Empresa A difere das outras duas pelo fato de que seus diretores têm um perfil de formação empresarial e domínio de ferramentas de gestão claramente superiores. Até que ponto isso impacta na estruturação de processos mais bem orientados à gestão de riscos é um ponto que fica em aberto.

No que diz respeito às atividades de projeto propriamente ditas, o planejamento do processo de projeto, a coordenação das disciplinas projetuais e a determinação de soluções e interface são consideradas pelas empresas questões operacionais de menor importância do ponto de vista de impacto na gestão do custo e prazo de entrega dos empreendimentos.

A Empresa A é, de certa forma, uma exceção, na medida em que vem implantando de maneira sistemática melhorias no processo de coordenação de projetos.

Como observado na Tabela 3, em geral as empresas não fazem orçamentos ou planejamento das obras considerando índices históricos de produtividade, o que implica aumento da incerteza e risco. Os resultados apontam para o fato de que a falta de integração entre as etapas de projeto e execução das obras faz com que orçamentos e cronogramas físicos e financeiros contenham imprecisões e até mesmo erros, introduzindo mais incerteza no processo de planejamento da execução.

\section{Critérios para priorização de atividades de risco identificadas no estudo de caso}

Uma vez identificadas as atividades associadas à gestão de riscos, uma questão que se coloca é: quais dessas atividades tem maior impacto na redução da incerteza (risco) no processo de projeto? Colocando de outra forma, assumindo que haja um conjunto de atividades que, mesmo implicitamente, estejam associadas à gestão de risco, quais são as mais relevantes?

A questão é importante na medida em que uma priorização de atividades permitirá a definição de diretrizes para a melhoria do processo de gestão de riscos na fase de concepção e projeto dos empreendimentos.

Podem ser definidas quais atividades deverão ser necessariamente conduzidas ou quais atividades são recomendáveis. Por outro lado, atividades mais relevantes, se destacadas, poderão ser mais bem explicitadas, padronizadas e pouco a pouco incorporadas à rotina do processo de projeto.
Com esse objetivo, foram identificadas atividades de maior relevância para a gestão de riscos com base no referencial apresentado no Project Definition Rating Index - Buildings (PDRI), apresentado anteriormente.

Foi definida uma correlação entre os processos do macrofluxo de processos das empresas, conforme sintetizados na Tabela 3 , com os respectivos elementos do PDRI, com seus pesos discriminados. Essa correlação é apresentada na Tabela 4.

Assume-se que processos das empresas correspondam a processos do PDRI, e a eles sejam atribuídos os pesos máximos. Uma comparação entre pesos permite identificar ações com maior impacto na gestão de riscos.

Assim, a atividade ligada à prospecção de terrenos "pesquisas online", correspondente à fase de prospecção de terrenos, está associada, no PDRI, ao processo "requisitos para instalação", representando 31 pontos na escala geral. Ou seja, assumindo que a atividade não é bem executada, seu impacto no aumento do risco de insucesso é pequeno quando comparada a outras atividades como, por exemplo, "desenvolvimento dos projetos executivos", que é bem mais crítica, com peso 158.

Como é possível observar na tabela anterior, algumas atividades se repetem em diferentes processos. Isso se deve ao fato de que mais de um processo cumpre o objetivo esperado pela atividade proposta pelo CII. Por isso, a soma das pontuações supera o valor de teto estabelecido pelo PDRI, 1000. No entanto, isso não afeta a análise, visto que, neste caso, o PDRI está sendo aplicado visando identificar os processos mais ou menos críticos no processo de concepção de empreendimentos imobiliários.

Atividades de maior peso foram definidas como "mandatórias", outras são definidas como "recomendáveis", e há um grupo de atividades que necessariamente serão executadas, ou seja, a identificação e/ou análise de risco é implícita a uma atividade necessariamente executada nos processos de incorporação e projeto. Essas atividades são identificadas como "intrínseco" ao processo de incorporação e/ou projeto. Dessa forma, estabelece-se uma hierarquia de atividades em função de seu impacto em termos de risco.

Se considerarmos as atividades do processo de projeto hierarquizadas tomando como referência o peso que as atividades correspondentes a elas têm no PDRI, observamos que as duas atividades de maior impacto no tratamento de riscos estão associadas ao desenvolvimento dos projetos 
arquitetônicos e complementares, atividades essas negligenciadas pelos empreendedores.

A atividade "desenvolvimento dos projetos executivos" diz respeito às soluções de projeto e interfaces entre projetos arquitetônicos e complementares, ou seja, aspectos diretamente associados à coordenação de projetos.
Nota-se que essa atividade só é executada de uma maneira satisfatória pela Empresa A, que estabeleceu um sistema de gestão do processo de projeto. As demais empresas parecem desenvolver essa atividade de maneira reativa, ou seja, resolvendo problemas de interfaces entre projetos à medida que aparecem.

\section{Tabela 4 - Agrupamento de atividades em função de sua relevância}

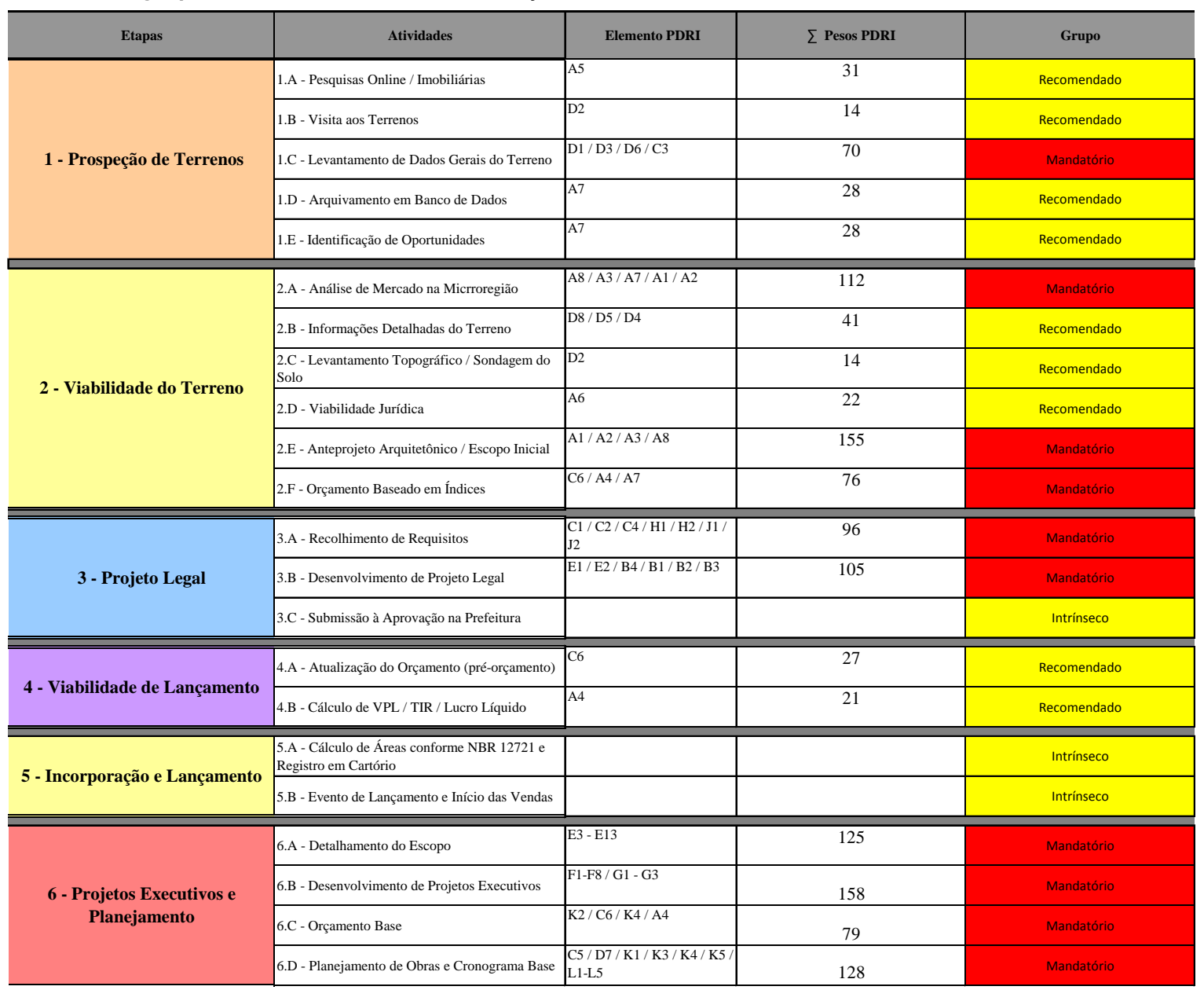

\section{Discussão}

Em função das análises das atividades relacionadas com a gestão de riscos desenvolvidas no processo de projeto, sintetizadas na Tabela 3, assim como quanto a sua priorização de acordo com os critérios propostos pelo PDRI, explicitada na Tabela 4, alguns comentários podem ser feitos.

Nas fases iniciais de concepção, verificou-se que maior atenção é concentrada no estudo de viabilidade econômica, em detrimento de outros aspectos.

A definição do programa de necessidades e a formalização do escopo dos empreendimentos são vistas como atividades de menor importância, talvez pelo fato de que no nicho de mercado dessas empresas os produtos são mais repetidos e padronizados. Nos casos apresentados, somente a Empresa A faz estudos alternativos para a concepção de seus projetos, verificando entre as alternativas uma solução ótima.

Independentemente de sua maturidade gerencial, as empresas têm, do ponto de vista da gestão de riscos, várias características em comum, além do fato de não estabelecerem em quase todas as etapas do macrofluxo de projetos ações formalmente orientadas à gestão de riscos. 
Um primeiro aspecto refere-se ao fato de que a identificação de riscos é mais explícita na análise de viabilidade do terreno e na análise econômicofinanceira dos empreendimentos.

À medida que as etapas se desenvolvem, parece diminuir a importância dada pelas empresas à identificação e tratamento de riscos. Esse mesmo comportamento foi encontrado em trabalhos citados anteriormente (MC GRAW-HILL, 2014; ROSTAMI et al., 2015): em empresas incorporadoras de médio porte, a análise de riscos concentra sua atenção em aspectos associados à rentabilidade dos empreendimentos, e menos em questões associadas ao processo de definição do produto e do planejamento de sua execução.

Um dos desdobramentos disso é o fato de que a atividade projetual, nas fases de desenvolvimento de projetos básicos e executivos, tem pouco impacto na gestão de riscos. As empresas não têm mecanismos estruturados para tratamento das mudanças no escopo dos projetos, ou para a gestão da informação ao longo das mudanças que ocorrem em função das dependências e interfaces entre projetos. Nesse sentido, espera-se que a introdução de um modelo de coordenação de projetos e a introdução de mecanismos de gestão da informação tornem mais explícitos riscos e incertezas, e contribuam para a mitigação de riscos nesse processo de projeto, na mesma direção apontada pelos trabalhos citados acima.

Nota-se nas três empresas que pelo fato de as ações para gestão de riscos serem pouco explicitadas nos processos de incorporação e projeto, duas limitações se apresentam de maneira recorrente nos empreendimentos. Por um lado, há dificuldade de registro e reflexão sobre "lições aprendidas" na gestão de riscos.

Configura-se um ciclo vicioso, em que as empresas agem reativamente e, de um empreendimento para outro, não incorporam mecanismos para quantificação da probabilidade de ocorrência de falhas ou de seu impacto sobre os resultados do empreendimento. Quando isso é feito, acontece de maneira empírica, dependendo da expertise dos agentes envolvidos nos processos a cada momento.

Por outro lado, há dificuldade de priorização de riscos que permita alocar recursos para sua gestão de maneira eficaz. Esse aspecto vem sendo apontado na literatura recente como comum a empresas de médio porte com características similares (ROSTAMI et al., 2015).

Percebe-se, por exemplo, que as verbas alocadas a contingenciamento são definidas de maneira empírica, possivelmente pelo fato de que os gestores julgam ter segurança com relação ao potencial custo das obras. Nesse caso, o comportamento das empresas é similar ao reportado em outros trabalhos da literatura (MC GRAW-HILL, 2014).

Do ponto de vista da priorização das atividades, são associadas à fase de desenvolvimento dos projetos atividades consideradas críticas, fase esta negligenciada pelas empresas. Esse comportamento sugere que a gestão do processo de projeto não é vista como condição de redução de riscos do empreendimento.

$\mathrm{Na}$ mesma linha de raciocínio, a atividade "anteprojeto arquitetônico - escopo inicial" também é crítica no tratamento de riscos e não é realizada de maneira satisfatória pelas empresas. Possivelmente a pouca importância dada aos estudos iniciais e definição do anteprojeto arquitetônico esteja associada ao fato de que o desenvolvimento de projetos ainda é visto exclusivamente como um custo, e não como um fator fundamental para o sucesso dos empreendimentos. Uma evidência disso é o fato de que vários empreendimentos das três empresas os anteprojetos, desenvolvidos externamente, foram contratados utilizando o critério do menor preço.

A hierarquização de atividades aponta também para o fato de que mecanismos de coordenação de projeto e análise de soluções de interface entre disciplinas de projeto precisam ser mais bem elaborados nas empresas. Nesse sentido, os sistemas de gestão da qualidade ainda não implicaram melhorias efetivas no processo de projeto. A coordenação de projetos deveria ser uma atividade mais sinérgica com as funções associadas à incorporação e à modelagem de negócio, nas quais a percepção de potenciais riscos é mais bem concretizada.

Da mesma forma, a atividade "planejamento da obra e cronograma base" também é negligenciada na etapa de projetos. De fato, nessas empresas o planejamento da produção fica a cargo dos engenheiros de produção, já na fase de execução dos empreendimentos.

Essas características apontam para o fato, a ser comprovado com análise de maior número de empresas e empreendimentos, de que empresas de médio porte ainda não incorporaram plenamente o conceito de priorizar as atividades de projeto como mecanismo de redução de incertezas na etapa de execução das obras.

\section{Conclusão}

O presente trabalho apresentou um mapeamento dos processos de incorporação, concepção e desenvolvimento de projetos de empreendimentos 
de construtoras de médio porte, associando esses processos aos mecanismos de identificação e tratamento de riscos. Nesse sentido, o referencial conceitual apresentado pela ISO 31000 e pelo PMBOK mostrou-se adequado, proporcionando elementos objetivos para a identificação das atividades no processo de projeto que impactam no tratamento de riscos.

A exemplo do que foi reportado na literatura internacional, observou-se que as empresas não têm mecanismos formais e explícitos para gerenciamento de riscos. Quando isso acontece, esses mecanismos estão mais concentrados nas etapas iniciais de análise de viabilidade técnica, jurídica e econômica dos empreendimentos.

Nas empresas estudadas, o processo de projeto é negligenciado: não existem mecanismos formais de coordenação de projetos, controle de mudanças e análise estruturada de soluções projetuais para as interfaces entre projetos. Nesse sentido, os sistemas de gestão da qualidade parecem ainda não influir efetivamente na gestão do processo de projeto, e desde o ponto de vista de seu desenvolvimento os sistemas voltam-se mais para garantir que os requisitos normativos para a certificação sejam atendidos.

A hierarquização de atividades do processo de projeto do ponto de vista de seu impacto no aumento da incerteza e risco dos empreendimentos, usando os critérios propostos pelo Project Definition Rating Index, aponta para o fato de que o desenvolvimento do programa de necessidades, a definição do escopo dos projetos, o desenvolvimento do anteprojeto e o detalhamento dos projetos executivos - quando é feita a compatibilização final entre as disciplinas de projeto - são atividades críticas do ponto de vista de risco. Por serem negligenciadas, tem-se aqui uma clara oportunidade de introdução de melhorias no próprio processo de projeto, com impacto direto na gestão de riscos.

A explicitação das atividades no processo de projeto ligadas à gestão de riscos torna-se importante na medida em que é um primeiro passo para priorizar ações voltadas ao tratamento de riscos e, como fruto de processo evolutivo, passa a ser viável implementar medidas sistêmicas de gestão de riscos, inseridas no processo de projeto das empresas.

\section{Referências}

ASSOCIAÇÃO BRASILEIRA DE NORMAS

TÉCNICAS. NBR ISO 31000: gestão de riscos: princípios e diretrizes. Rio de Janeiro, 2009.
AZEVEDO, R. Um modelo para gestão de risco na incorporação de imóveis usando a metodologia multicritério para apoio a decisão construtivista (MCDA-C). 2013. Tese (Doutorado em Engenharia Civil) - Escola de Engenharia, Universidade Federal de Santa Catarina, Florianópolis, 2013.

BARRETO, F. Contribuição à análise de riscos no processo de projeto de empreendimentos imobiliários. 2015. 226 f. Dissertação (Mestrado em Construção Civil) - Escola de Engenharia, Universidade Federal de Minas Gerais, Belo Horizonte, 2015.

BHARANTY, G.; MCSHANE, M. Applying a System Model to Entreprise Risk Management. Engineering Management Journal, v. 26, n. 4, p. 38-47, 2014.

BICALHO F. Sistema de Gestão da Qualidade para Empresas Construtoras de Pequeno Porte. Belo Horizonte, 2009. 147 f. Dissertação (Mestrado em Construção Civil) - Escola de Engenharia, Universidade Federal de Minas Gerais, Belo Horizonte, 2009.

BUZI, D.; JUNGLES, E.; AVILA, A.

Gerenciamento de Riscos em Incorporações Imobiliárias: uma abordagem utilizando a lógica fuzzy. In: ENCONTRO NACIONAL DE TECNOLOGIA DO AMBIENTE CONSTRUÍDO, 14., Juiz de Fora, 2012. Anais... Juiz de Fora: ANTAC, 2012.

CARDOSO, F. et al. Public Policy Instruments to Encourage Construction Innovation: Overview of the Brazilian Case. In: MANSEAU, A.; SEADEN, G. (Ed.). Innovation in construction: an international review of public policies. London: Spon Press, 2012.

CÂMARA BRASILEIRA DA INDÚSTRIA DA CONSTRUÇÃO. Boletim Estatístico 2015.

Disponível em:

<http://www.cbicdados.com.br/menu/indicadoreseconomicos-gerais/>. Acesso em: 26 fev. 2015.

CHAPMAN, R. The Controlling Influences on Effective Risk Identification and Assessment For Construction Design. International Journal of Project Management, London, v. 19, n. 3, p. 147 160, 2001.

CHO, C.; GIBSON, E. Building Project Scope Definition Using Project Definition Rating Index. Journal of Architectural Engineering, v. 12, n. 1, p. 115-125, 2001.

CONSTRUCTION INDUSTRY INSTITUTE. Project Definition Rating Index: building projects. Austin: University of Texas at Austin, 2008. Implementation Report 155-2. 
EDWARDS, P.; BOWEN, P. Risk and Risk Management in Construction: a review and future directions for research. Engineering, Construction and Architectural Management, London, v. 5, n. 4, p. 339-349, 1998.

EMMIT, S.; DEMIAN, P.; ALHARBI, M. Architectural Management: exploring definitions and impacts. In: CIB W096 MEETING: ARCHITECTURAL MANAGEMENT IN THE DIGITAL ARENA, Viena, 2011. Proceedings.... Viena: CIB, 2011.

FAGEHA, M.; AIBINU, A. Prioritizing Project Scope Definition Elements in Public Building Projects. Australian Journal of Construction Economics and Building, v. 4, n. 3, p. 18-33, 2014.

GEOFREY, P.; HALCROW, W. Control of Risk: a guide to systematic management of risk from construction. CIRIA Special Publication 125, 1996.

HUFFMANN, F. Corporate Real Estate Risk Management and Assessment. Journal of Corporate Real Estate, v. 5, n. 1, p. 32-41, 2002.

MC GRAW-HILL, Managing Uncertainty and Expectations in Building Design and Construction. McGraw-Hill Construction Report 2014.

MARQUES, U.; STARLING, C.; ANDERY, P. Gestão de Fatores de Risco e Incerteza que Influenciam o Sucesso dos Empreendimentos de Incorporação Imobiliária Aplicando o Project Definition Rating Index. In: SIMPÓSIO BRASILEIRO DE QUALIDADE DO PROJETO, Campinas, 2013. Anais... Campinas: ANTAC, 2013.
PRINS, M.; OWEN, R. Integrated Delivery and Design Solutions.Architectural Engineering and Design Management, London, v. 6, n. 4, p. 227231, 2010.

\section{PROJECT MANAGEMENT INSTITUTE.}

Project Management Body of Knowledge, Newtown Square (USA): Project Management Institute, 2013.

\section{PROJECT MANAGEMENT INSTITUTE. Practice Standard for Project Risk \\ Management. Newtown Square: Project Management Institute, 2009.}

PURDY, G. ISO31000:2009: setting an standard for risk management. Risk Analysis, v. 30, n. 6, p. 881-886, 2010.

ROSTAMI, A. et al. Risk Management in Small and Medium Entreprises in the UK Construction Industry. Engineering Construction and Architectural Management, London, v. 22, n. 1, p. 91-107, 2015.

SABADA, S. et al. Project Risk Management Methodology for Small Firms. International Journal of Project Management, London, v. 32, n. 1, p. 327-340, 2014.

SOUZA, V.; ALMEIDA, N.; DIAS, L. Risk Management Framework For the Construction Industry According to ISO31000:2009. Journal of Risk Analysis and Crisis Response, v. 2, n. 4, p. 261-274, 2012.

TAROUN, A. Towards a Better Modelling and Assessment of Construction Risk: insights from literature review. International Journal of Project Management, London, v. 32, n. 1, p. 101115, 2014.

YIN, R. Case Study Research: design and methods. 5. ed. London: Sage, 2013.

Felipe de Souza Pinto Barreto

Departamento de Engenharia de Materiais e Construção Civil, Escola de Engenharia | Universidade Federal de Minas Gerais | Av. Presidente Antônio Carlos, 6627, Bloco I, Sala 3314, São José | Belo Horizonte - MG - Brasil | CEP 31275-013 | Tel.: (31) 3409-1856 | E-mail: felipebarreto87@gmail.com

Paulo Roberto Pereira Andery

Departamento de Engenharia de Materiais e Construção Civil, Escola de Engenharia | Universidade Federal de Minas Gerais |

E-mail: paulo@demc.ufmg.br

Revista Ambiente Construído

Associação Nacional de Tecnologia do Ambiente Construído

Av. Osvaldo Aranha, $99-3^{\circ}$ andar, Centro

Porto Alegre - RS - Brasil

CEP 90035-190

Telefone: +55 (51) 3308-4084

Fax: +55 (51) 3308-4054

www.seer.ufrgs.br/ambienteconstruido

E-mail: ambienteconstruido@ufrgs.br 